\title{
A conservative difference scheme for the Riesz space-fractional sine-Gordon equation
}

\section{Zhiyong Xing ${ }^{1,2^{*}}$ (D) and Liping Wen ${ }^{1}$}

"Correspondence:

201690110064@smail.xtu.edu.cn

${ }^{1}$ School of Mathematics and Computational Science, Xiangtan

University, Xiangtan, P.R. China ${ }^{2}$ Department of Mathematics,

Shaoyang University, Shaoyang, P.R. China

\begin{abstract}
In this paper, we study a conservative difference scheme for the sine-Gordon equation (SGE) with the Riesz space fractional derivative. We rigorously establish the conservation property and solvability of the difference scheme. We discuss the stability and convergence of the difference scheme in the $L_{\infty}$ norm. To reduce the computational complexity, we introduce a revised Newton method for implementing the difference scheme. Finally, we provide several numerical experiments to support the theoretical results.
\end{abstract}

Keywords: Space-fractional sine-Gordon equation; Riesz fractional derivative; Conservation law; Newton method; Convergence and stability

\section{Introduction}

The nonlinear SGE

$$
\frac{\partial^{2} u}{\partial t^{2}}-\Delta u+\sin u=0, \quad \mathbf{x}=\left(x_{1}, \ldots, x_{n}\right) \in \mathbb{R}^{n}
$$

arises in many different areas, such as stability of fluid motions, differential geometry, Josephson junctions, models of particle physics [1], the propagation of fluxon [2], the motion of a rigid pendulum attached to a stretched wire [3], the phenomenon of supratransmission in nonlinear media [4], and so on. Therefore the investigation for the SGE has attracted attention of some researchers, and many significant achievements have been made.

A remarkable property of (1.1) is the energy conservation:

$$
\frac{1}{2} \int_{\mathbb{R}}\left[\left(\frac{\partial u}{\partial t}\right)^{2}+\left(\frac{\partial u}{\partial x}\right)^{2}+2 P(u)\right] d x=\text { const }
$$

where $P(u)=1-\cos u$; it was studied by many researchers [5-9]. The conserved quantity is good for the analysis of the nonlinear stability of the numerical schemes proposed, although it is difficult to apply them $[10,11]$.

Since the fractional calculus is frequently better than the integer calculus in the description of many physical laws, various classical partial differential equations have been extended to the corresponding fractional-order differential equations [12-19]. However,

(c) The Author(s) 2018. This article is distributed under the terms of the Creative Commons Attribution 4.0 International License (http://creativecommons.org/licenses/by/4.0/), which permits unrestricted use, distribution, and reproduction in any medium, provided you give appropriate credit to the original author(s) and the source, provide a link to the Creative Commons license, and indicate if changes were made. 
there are a few works on fractional-order SGEs. Ray [20] combined the modified decomposition method and Fourier transform to approximate the solution of a fractional SGE. In [21], a family of breather-like solutions for the fractional SGE is found numerically by using the approach that is called sometimes the rotating wave approximation. MacíasDíaz [4] employed an explicit finite difference scheme to simulate a space-fractional SGE. His result supported the fact that nonlinear supratransmission is present in the Riesz space-fractional model. He also pointed out that numerical simulations for fractional SGE require enormous amount of computer time.

In this paper, we consider the space-fractional SGE

$$
\frac{\partial^{2} u}{\partial t^{2}}-\frac{\partial^{\alpha} u}{\partial|x|^{\alpha}}+\sin u=0, \quad-\infty<x<+\infty, 0<t \leq T,
$$

with the boundary and initial conditions

$$
\begin{array}{ll}
u(x, 0)=\varphi(x), & \frac{\partial u(x, 0)}{\partial t}=\psi(x), \quad-\infty<x<+\infty \\
\lim _{|x| \rightarrow \infty} u(x, t)=0, & 0<t<T
\end{array}
$$

where $1<\alpha \leq 2$.

The Riesz fractional derivative of order $\alpha$ is defined by [22]

$$
\frac{\partial^{\alpha} u}{\partial|x|^{\alpha}}(x, t)=-(-\Delta)^{\frac{\alpha}{2}} u(x, t)=-\frac{1}{2 \cos (\pi \alpha / 2)}\left({ }_{-\infty} D_{x}^{\alpha} u(x, t)+{ }_{x} D_{+\infty}^{\alpha} u(x, t)\right),
$$

where ${ }_{-\infty} D_{x}^{\alpha} u(x, t)$ and ${ }_{x} D_{+\infty}^{\alpha} u(x, t)$ are the left- and right-side Riemann-Liouville fractional derivatives, respectively.

Multiplying (1.2) by $\frac{\partial u}{\partial t}$ and then integrating with respect to $x$, we obtain

$$
\frac{d}{d t} \int_{\mathbb{R}} \frac{1}{2}\left[\left(\frac{\partial u}{\partial t}\right)^{2}+\left((-\triangle)^{\frac{\alpha}{4}} u\right)^{2}+2 P(u)\right] d x=0,
$$

that is, the conservation of energy

$$
\frac{1}{2}\left\|\frac{\partial u}{\partial t}\right\|_{L^{2}}^{2}+\frac{1}{2}\left\|(-\triangle)^{\frac{\alpha}{4}} u\right\|_{L^{2}}^{2}+\int_{\mathbb{R}} P(u) d x=\text { const },
$$

where $P(u)$ is consistent with the aforementioned.

To the best of our knowledge, there are very few works developing conservative numerical methods for fractional SGEs. The main objective of this paper is to propose a conservative numerical method for space-fractional SGEs and try to reduce the computational complexity.

This paper is arranged as follows. In the next section, we propose a conservative difference scheme for space-fractional SGEs. Subsequently, we prove that the difference scheme preserves the energy conservation law. The boundedness, solvability, and convergence of the difference scheme are rigorously established. In Sect. 4, we introduce a revised Newton method for implementation of the difference scheme. In Sect. 5, we present some numerical results to demonstrate the effectiveness of the difference scheme. Finally, we give a simple conclusion. 


\section{A conservative difference scheme for fractional SGEs}

\subsection{Notation}

To develop a finite difference scheme for problem (1.2)-(1.4), we assume that its solution is negligibly small outside of the interval $\Omega=\left(x_{R}, x_{L}\right)$, that is, $\left.u\right|_{x \in \mathbb{R} \backslash \Omega}=0$. we choose the time step $\tau=\frac{T}{N}$ and mesh size $h=\frac{x_{R}-x_{L}}{M}$ with two positive integers $N$ and $M$. Denote

$$
\Omega_{h}=\left\{x_{i} \mid x_{i}=x_{L}+i h, 1 \leq i \leq M-1\right\}, \quad \Omega_{\tau}=\left\{t_{n} \mid t_{n}=n \tau, 0 \leq n \leq N\right\}
$$

and

$$
u_{i}^{n} \approx u\left(x_{i}, t_{n}\right), \quad U_{i}^{n}=u\left(x_{i}, t_{n}\right) .
$$

Let $v_{h}=\left\{w \mid w=\left(w_{1}, w_{2}, \ldots, w_{M-1}\right)^{T}\right\}$ be the space of grid functions. For a given grid function $w=\left\{w_{i}^{n} \mid\left(x_{i}, t_{n}\right) \in \Omega_{h} \times \Omega_{\tau}\right\}$, we define the finite difference operators

$$
\begin{aligned}
\left(w_{i}^{n}\right)_{t}=\frac{w_{i}^{n+1}-w_{i}^{n}}{\tau}, & \left(w_{i}^{n}\right)_{\bar{t}}=\frac{w_{i}^{n}-w_{i}^{n-1}}{\tau}, \\
\left(w_{i}^{n}\right)_{\hat{t}}=\frac{w_{i}^{n+1}-w_{i}^{n-1}}{2 \tau}, & \bar{w}_{i}^{n}=\frac{w_{i}^{n+1}+w_{i}^{n-1}}{2} .
\end{aligned}
$$

For any two grid functions $u^{n}$ and $v^{n}$, we define

$$
\left(u^{n}, v^{n}\right)=h \sum_{i=1}^{M-1} u_{i}^{n} v_{i}^{n}, \quad\left\|u^{n}\right\|^{2}=\left(u^{n}, u^{n}\right), \quad\left\|u^{n}\right\|_{l_{h}^{\infty}}=\sup _{i \in \mathbb{Z}}\left|u_{i}\right| .
$$

Let $0 \leq \sigma \leq 1$ be given. For any $u \in l_{h}^{2}$, the fractional Sobolev norm $\|u\|_{H^{\sigma}}$ and seminorm $|u|_{H^{\sigma}}$ can be defined as

$$
\|u\|_{H^{\sigma}}^{2}=h \int_{-\pi}^{\pi}\left(1+h^{-2 \sigma}|\mathbf{k}|^{2 \sigma}\right)|\widehat{u}(\mathbf{k})|^{2} d \mathbf{k}, \quad|u|_{H^{\sigma}}^{2}=h \int_{-\pi}^{\pi} h^{-2 \sigma}|\mathbf{k}|^{2 \sigma}|\widehat{u}(\mathbf{k})|^{2} d \mathbf{k} .
$$

\subsection{A conservative implicit difference scheme}

Lemma 2.1 ([23]) Suppose that $u \in \mathcal{L}^{2+\alpha}(\mathcal{R})$. Then

$$
-h^{-\alpha} \triangle_{h}^{\alpha} f(x)=-(-\triangle)^{\frac{\alpha}{2}} f(x)+O\left(h^{2}\right)
$$

where

$$
\Delta_{h}^{\alpha} f(x)=\sum_{k=-\infty}^{\infty} g_{k}^{(\alpha)} f(x-k h), \quad g_{k}^{(\alpha)}=\frac{(-1)^{k} \Gamma(\alpha+1)}{\Gamma(\alpha / 2-k+1) \Gamma(\alpha / 2+k+1)}
$$

If we define

$$
u^{*}(x, t)= \begin{cases}u(x, t) & \text { if } x \in\left[x_{L}, x_{R}\right] \\ 0 & \text { if } x \in\left(-\infty, x_{L}\right) \cup\left(x_{R}, \infty\right)\end{cases}
$$


then, for $1<\alpha \leq 2$, we can get

$$
-(-\triangle)^{\frac{\alpha}{2}} u_{i}^{n}=-h^{-\alpha} \sum_{l=1}^{M-1} g_{i-l}^{(\alpha)} u_{l}^{n}+O\left(h^{2}\right)
$$

Denote

$$
\delta_{h}^{\alpha} u_{i}^{n}=h^{-\alpha} \sum_{l=1}^{M-1} g_{i-l}^{(\alpha)} u_{l}^{n}
$$

Considering equation (1.2) at points $\left(x_{i}, t_{n}\right)$, we derive

$$
\frac{\partial^{2} u}{\partial t^{2}}\left(x_{i}, t_{n}\right)-\frac{\partial^{\alpha} u}{\partial|x|^{\alpha}}\left(x_{i}, t_{n}\right)=-\sin u\left(x_{i}, t_{n}\right), \quad 1 \leq i \leq M-1,1 \leq n \leq N-1
$$

Combining the Taylor expansion and equation (2.3), we get

$$
\begin{aligned}
& \frac{\partial^{2} u}{\partial t^{2}}\left(x_{i}, t_{n}\right)=\left(U_{i}^{n}\right)_{t \bar{t}}+O\left(\tau^{2}\right), \\
& \frac{\partial^{\alpha} u}{\partial|x|^{\alpha}}\left(x_{i}, t_{n}\right)=\delta_{h}^{\alpha} \bar{U}_{i}^{n}+O\left(h^{2}+\tau^{2}\right), \\
& -\sin u\left(x_{i}, t_{n}\right)=\zeta\left(\bar{U}_{i}^{n}\right)+O\left(\tau^{2}\right),
\end{aligned}
$$

where $\zeta\left(\bar{U}_{i}^{n}\right)=\frac{\cos \left(U_{i}^{n+1}\right)-\cos \left(U_{i}^{n-1}\right)}{U_{i}^{n+1}-U_{i}^{n-1}}$.

Substituting (2.5) into (2.4), we get

$$
\left(U_{i}^{n}\right)_{t \bar{t}}+\delta_{h}^{\alpha} \bar{U}_{i}^{n}=\zeta\left(\bar{U}_{i}^{n}\right)+r_{i}^{n}
$$

where $r_{i}^{n}=O\left(h^{2}+\tau^{2}\right)$

In addition, from conditions (1.3) and (1.4) we have

$$
\begin{aligned}
& U_{0}^{n}=0, \quad U_{M}^{n}=0, \quad 0 \leq n \leq N, \\
& U_{i}^{0}=\varphi\left(x_{i}\right), \quad \frac{\partial U}{\partial t}\left(x_{i}, 0\right)=\psi\left(x_{i}\right), \quad 1 \leq i \leq M-1 .
\end{aligned}
$$

Omitting the small term $r_{i}^{n}$ in (2.6) and using the numerical solution $u_{i}^{n}$ to replace $U_{i}^{n}$, we obtain the following difference scheme for solving problem (1.2)-(1.4):

$$
\begin{aligned}
& \left(u_{i}^{n}\right)_{t \bar{t}}+\delta_{h}^{\alpha} \bar{u}_{i}^{n}=\zeta\left(\bar{u}_{i}^{n}\right), \quad 1 \leq i \leq M-1,1 \leq n \leq N-1, \\
& u_{0}^{n}=0, \quad u_{M}^{n}=0, \quad 0 \leq n \leq N, \\
& u_{i}^{0}=\varphi\left(x_{i}\right), \quad u_{i}^{1}=u_{i}^{0}+\tau \psi\left(x_{i}\right)-\frac{\tau^{2}}{2}\left[\delta_{h}^{\alpha} u_{i}^{0}+\sin \left(u_{i}^{0}\right)\right], \quad 1 \leq i \leq M-1 .
\end{aligned}
$$




\section{Numerical analysis}

\subsection{Some useful lemmas}

Lemma 3.1 ([13]) If $u, v$ are any two grid functions in $v_{h}$, then there exists a linear operator $\Lambda_{h}^{\alpha}=h^{-\frac{\alpha}{2}} G^{\frac{1}{2}}$ such that

$$
\left(\delta_{h}^{\alpha} u, v\right)=\left(h^{-\alpha} G u, v\right)=\left(\Lambda_{h}^{\alpha} u, \Lambda_{h}^{\alpha} v\right)
$$

where

$$
G=\left(\begin{array}{ccc}
g_{0}^{(\alpha)} & g_{-1}^{(\alpha)} & \ldots g_{-M+2}^{(\alpha)} \\
g_{1}^{(\alpha)} & g_{0}^{(\alpha)} & \ldots g_{-M+3}^{(\alpha)} \\
\vdots & \vdots & \ddots \vdots \\
g_{M-2}^{(\alpha)} & g_{M-3}^{(\alpha)} & \cdots g_{0}^{(\alpha)}
\end{array}\right) ;
$$

$G^{\frac{1}{2}}$ is the unique positive definite square root of $G$, that is, $\left(G^{\frac{1}{2}}\right)^{2}=G$. Moreover, we have

$$
\left(\delta_{h}^{\alpha} u, u\right)=\left(\Lambda_{h}^{\alpha} u, \Lambda_{h}^{\alpha} u\right)=\left\|\Lambda_{h}^{\alpha} u\right\|^{2},
$$

where $\delta_{h}^{\alpha} u=\left(\delta_{h}^{\alpha} u_{1}, \delta_{h}^{\alpha} u_{2}, \ldots, \delta_{h}^{\alpha} u_{M-1}\right)^{T}$.

Lemma 3.2 If $u^{n} \in v_{h}$, then we have

$$
\begin{aligned}
& \left(u_{t \bar{t}}^{n}, 2 u_{\hat{t}}^{n}\right)=\left(\left\|u_{t}^{n}\right\|^{2}\right)_{\bar{t}}, \\
& \left(\delta_{h}^{\alpha} \bar{u}^{n}, 2 u_{\hat{t}}^{n}\right)=\left\|\Lambda_{h}^{\alpha} u^{n}\right\|_{\hat{t}}^{2} .
\end{aligned}
$$

Proof Equality (3.2) can be found in [8], so we omit it here. Equality (3.3) can be proved as follows:

$$
\begin{aligned}
\left(\delta_{h}^{\alpha} \bar{u}^{n}, 2 u_{\hat{t}}^{n}\right) & =\frac{1}{2 \tau}\left(\delta_{h}^{\alpha} u^{n+1}+\delta_{h}^{\alpha} u^{n-1}, u^{n+1}-u^{n-1}\right) \\
& =\frac{1}{2 \tau}\left[\left(\delta_{h}^{\alpha} u^{n+1}, u^{n+1}\right)-\left(\delta_{h}^{\alpha} u^{n-1}, u^{n-1}\right)+\left(\delta_{h}^{\alpha} u^{n-1}, u^{n+1}\right)-\left(\delta_{h}^{\alpha} u^{n+1}, u^{n-1}\right)\right] \\
& =\frac{1}{2 \tau}\left[\left(\delta_{h}^{\alpha} u^{n+1}, u^{n+1}\right)-\left(\delta_{h}^{\alpha} u^{n-1}, u^{n-1}\right)\right] \\
& =\left\|\Lambda_{h}^{\alpha} u^{n}\right\|_{\hat{t}}^{2} .
\end{aligned}
$$

The proof ends.

Lemma 3.3 ([8]) Let $\omega(k)$ and $\rho(k)$ be nonnegative mesh functions. If $C>0, \rho(k)$ is nondecreasing, and

$$
\omega(k) \leq \rho(k)+C \tau \sum_{l=0}^{k-1} \omega(l)
$$

for all $k$, then

$$
\omega(k) \leq \rho(k) e^{C k \tau}
$$

for all $k$. 
Lemma 3.4 (Discrete Sobolev inequality [24]) For every $\frac{1}{2}<\sigma \leq 1$, there exists a constant $C=C(\sigma)>0$ independent of $h>0$ such that

$$
\|u\|_{l_{h}^{\infty}} \leq C\|u\|_{H^{\sigma}}
$$

for all $u \in l_{h}^{2}$.

Lemma 3.5 (Uniform norm equivalence [25]) For any grid function $u=\left\{u_{j}\right\}$ and every $1<\alpha \leq 2$, we have

$$
\left(\frac{2}{\pi}\right)^{\alpha}|u|_{H^{\alpha / 2}}^{2} \leq\left(h^{-\alpha} \Delta_{h}^{\alpha} u, u\right) \leq|u|_{H^{\alpha / 2}}^{2} .
$$

Lemma 3.6 ([26], Lemma 1.4, Ch. 2) Let $X$ be a finite-dimensional Hilbert space with scalar product $[\cdot, \cdot]$ and norm $[\cdot]$, and let $\digamma$ be a continuous mapping from $X$ into itself such that

$$
[\digamma(\xi), \xi]>0 \text { for any }[\xi]=k>0
$$

Then there exists $\xi \in X$ with $[\xi] \leq k$ such that

$$
\digamma(\xi)=0 .
$$

\subsection{Conservation}

Theorem 3.1 The scheme (2.9)-(2.11) is conservative in the sense that

$$
\varepsilon^{n}=\varepsilon^{n-1}=\cdots=\varepsilon^{0},
$$

where

$$
\varepsilon^{n}=\frac{1}{2}\left\|u_{t}^{n}\right\|^{2}+\frac{1}{4}\left\|\Lambda_{h}^{\alpha} u^{n+1}\right\|^{2}+\frac{1}{4}\left\|\Lambda_{h}^{\alpha} u^{n}\right\|^{2}+\frac{h}{2} \sum_{i=0}^{M-1}\left[\left(1-\cos u_{i}^{n+1}\right)+\left(1-\cos u_{i}^{n}\right)\right]
$$

is the energy in the discrete sense.

Proof Making the discrete inner product of (2.9) with $2 u_{\hat{t}}^{n}$, we get that

$$
\left(\left(u^{n}\right)_{t \bar{t}}, 2 u_{\hat{t}}^{n}\right)+\left(\delta_{h}^{\alpha} \bar{u}^{n}, 2 u_{\hat{t}}^{n}\right)=\left(\zeta\left(\bar{u}^{n}\right), 2 u_{\hat{t}}^{n}\right)
$$

Directly computing, we have

$$
\begin{aligned}
\left(\zeta\left(\bar{u}^{n}\right), 2 u_{\hat{t}}^{n}\right)= & \frac{h}{\tau} \sum_{i=0}^{M-1}\left(\cos u_{i}^{n+1}-\cos u_{i}^{n-1}\right) \\
= & -\frac{h}{\tau} \sum_{i=0}^{M-1}\left\{\left[\left(1-\cos u_{i}^{n+1}\right)+\left(1-\cos u_{i}^{n}\right)\right]\right. \\
& \left.-\left[\left(1-\cos u_{i}^{n}\right)+\left(1-\cos u_{i}^{n-1}\right)\right]\right\} .
\end{aligned}
$$


Notice that other inner products in (3.4) can be calculated by Lemma 3.2. Substituting (3.2), (3.3), and (3.5) into (3.4), we obtain

$$
\begin{aligned}
& \left(\left\|u_{t}^{n}\right\|^{2}\right)_{\bar{t}}+\frac{1}{2 \tau}\left[\left(\left\|\Lambda_{h}^{\alpha} u^{n+1}\right\|^{2}+\left\|\Lambda_{h}^{\alpha} u^{n}\right\|^{2}\right)-\left(\left\|\Lambda_{h}^{\alpha} u^{n}\right\|^{2}+\left\|\Lambda_{h}^{\alpha} u^{n-1}\right\|^{2}\right)\right] \\
& \quad+\frac{h}{\tau} \sum_{i=0}^{M-1}\left\{\left[\left(1-\cos u_{i}^{n+1}\right)+\left(1-\cos u_{i}^{n}\right)\right]-\left[\left(1-\cos u_{i}^{n}\right)+\left(1-\cos u_{i}^{n-1}\right)\right]\right\} \\
& =0
\end{aligned}
$$

and hence

$$
\begin{aligned}
\varepsilon^{n} & =\frac{1}{2}\left\|u_{t}^{n}\right\|^{2}+\frac{1}{4}\left[\left(\left\|\Lambda_{h}^{\alpha} u^{n+1}\right\|^{2}+\left\|\Lambda_{h}^{\alpha} u^{n}\right\|^{2}\right)\right]+\frac{h}{2} \sum_{i=0}^{M-1}\left[\left(1-\cos u_{i}^{n+1}\right)+\left(1-\cos u_{i}^{n}\right)\right] \\
& =\frac{1}{2}\left\|u_{t}^{n-1}\right\|^{2}+\frac{1}{4}\left(\left\|\Lambda_{h}^{\alpha} u^{n}\right\|^{2}+\left\|\Lambda_{h}^{\alpha} u^{n-1}\right\|^{2}+\frac{h}{2} \sum_{i=0}^{M-1}\left[\left(1-\cos u_{i}^{n}\right)+\left(1-\cos u_{i}^{n-1}\right)\right],\right.
\end{aligned}
$$

that is,

$$
\varepsilon^{n}=\varepsilon^{n-1}=\cdots=\varepsilon^{0} .
$$

\subsection{The boundedness, solvability, and convergence}

Theorem 3.2 Assume that $\left\{u_{i}^{n} \mid 0 \leq i \leq M, 0 \leq n \leq N\right\}$ is a solution of the difference scheme (2.9)-(2.11). Then

$$
\left\|u_{t}^{n}\right\| \leq C, \quad\left\|\Lambda_{h}^{\alpha} u^{n}\right\| \leq C, \quad\left\|u^{n}\right\| \leq C, \quad\left\|u^{n}\right\|_{l_{h}^{\infty}} \leq C .
$$

Here and later, $C$ denotes a generic positive constant; in different places, it may represent different constants.

Proof By Theorem 3.1 we get

$$
2 \varepsilon^{n}=\left\|u_{t}^{n}\right\|^{2}+\frac{1}{2}\left[\left(\left\|\Lambda_{h}^{\alpha} u^{n+1}\right\|^{2}+\left\|\Lambda_{h}^{\alpha} u^{n}\right\|^{2}\right)\right]+h \sum_{i=0}^{M-1}\left[\left(1-\cos u_{i}^{n+1}\right)+\left(1-\cos u_{i}^{n}\right)\right]=C .
$$

Notice that

$$
\left(1-\cos u_{i}^{n+1}\right)+\left(1-\cos u_{i}^{n}\right) \geq 0 .
$$

Then

$$
\left\|u_{t}^{n}\right\| \leq C, \quad\left\|\Lambda_{h}^{\alpha} u^{n}\right\| \leq C .
$$

Moreover,

$$
\frac{\left\|u^{k+1}\right\|-\left\|u^{k}\right\|}{\tau} \leq\left\|u_{t}^{k}\right\| \leq C .
$$


By summing this equality for $k=0,1, \ldots, n-1(n \tau \leq T)$, we get

$$
\left\|u^{n}\right\| \leq n \tau C+\left\|u^{0}\right\| \leq C T+\left\|u^{0}\right\| \leq C .
$$

Combining Lemma 3.1 and Lemma 3.5, we derive

$$
\left|u^{n}\right|_{H^{\alpha / 2}}^{2} \leq\left(\frac{\pi}{2}\right)^{\alpha}\left(h^{-\alpha} \Delta_{h}^{\alpha} u^{n}, u^{n}\right)=\left(\frac{\pi}{2}\right)^{\alpha}\left(h^{-\alpha} G u^{n}, u^{n}\right)=\left(\frac{\pi}{2}\right)^{\alpha}\left\|\Lambda_{h}^{\alpha} u^{n}\right\|^{2} \leq C .
$$

Hence

$$
\left\|u^{n}\right\|_{H^{\alpha / 2}}^{2}=\left\|u^{n}\right\|^{2}+\left|u^{n}\right|_{H^{\alpha / 2}}^{2} \leq C .
$$

According to Lemma (3.4),

$$
\left\|u^{n}\right\|_{l_{h}^{\infty}} \leq C\left\|u^{n}\right\|_{H^{\alpha / 2}} \leq C
$$

Thus we get the desired results.

For the existence and uniqueness of a solution for the difference scheme (2.9)-(2.11), we have the following theorems.

Theorem 3.3 The difference scheme (2.9)-(2.11) has at least one solution.

Proof We carry out the proof by mathematical induction. Assume that $u^{0}, u^{1}, \ldots, u^{n}$ are given solutions. We aim to prove that there exists yet one $u^{n+1}$ satisfying the difference scheme. Let $X=v_{h}$ with the scalar product $(\cdot, \cdot)$ defined in (2.1). We define the mapping $\digamma: X \rightarrow X$ such that

$$
\begin{gathered}
(\digamma(\omega), v)=\left(\frac{\omega}{\tau^{2}}, v\right)-\left(\frac{2}{\tau} u_{t}^{n-1}, v\right)+\left(\frac{1}{2} \delta_{h}^{\alpha} \omega, v\right)+\left(\delta_{h}^{\alpha} u^{n-1}, v\right) \\
+\left(\frac{\cos u^{n-1}-\cos \left(\omega+u^{n-1}\right)}{\omega}, v\right), \quad \forall \omega, v \in X,
\end{gathered}
$$

where

$$
\begin{aligned}
& \frac{\cos u^{n-1}-\cos \left(\omega+u^{n-1}\right)}{\omega}=\left(\chi_{1}, \chi_{2}, \ldots, \chi_{M-1}\right)^{T}, \\
& \chi_{i}=\frac{\cos u_{i}^{n-1}-\cos \left(\omega_{i}+u_{i}^{n-1}\right)}{\omega_{i}}, \quad 1 \leq i \leq M-1 .
\end{aligned}
$$

Obviously, the mapping $\digamma$ is continuous. Moreover, we have

$$
\begin{aligned}
(\digamma(\omega), \omega)= & \frac{\|\omega\|^{2}}{\tau^{2}}-\frac{2}{\tau}\left(u_{t}^{n-1}, \omega\right)+\frac{1}{2}\left(\delta_{h}^{\alpha} \omega, \omega\right)+\left(\delta_{h}^{\alpha} u^{n-1}, \omega\right) \\
& +\left(\frac{\cos u^{n-1}-\cos \left(\omega+u^{n-1}\right)}{\omega}, \omega\right) .
\end{aligned}
$$


Applying Young's inequality and Theorem 3.2, we have

$$
\begin{aligned}
\frac{2}{\tau}\left(u_{t}^{n-1}, \omega\right) & =\left(2 \sqrt{2} u_{t}^{n-1}, \frac{1}{\sqrt{2} \tau} \omega\right) \\
& \leq 4\left\|u_{t}^{n-1}\right\|^{2}+\frac{1}{4} \frac{\|\omega\|^{2}}{\tau^{2}} \\
& \leq 8 \varepsilon^{0}+\frac{1}{4} \frac{\|\omega\|^{2}}{\tau^{2}}
\end{aligned}
$$

and

$$
\begin{aligned}
\left(\delta_{h}^{\alpha} u^{n-1}, \omega\right) & \leq \frac{\tau^{2}}{2}\left\|\delta_{h}^{\alpha} u^{n-1}\right\|^{2}+\frac{1}{2} \frac{\|\omega\|^{2}}{\tau^{2}} \\
& \leq 4 \tau^{2} h^{-\alpha} g_{0}^{(\alpha)} \varepsilon^{0}+\frac{1}{2} \frac{\|\omega\|^{2}}{\tau^{2}},
\end{aligned}
$$

where we used the fact that

$$
\begin{aligned}
\left\|\delta_{h}^{\alpha} u^{n-1}\right\|^{2} & =\left(h^{-\alpha} G u^{n-1}, h^{-\alpha} G u^{n-1}\right) \\
& \leq h^{-\alpha} \lambda_{\max }(G)\left(h^{-\alpha} G u^{n-1}, u^{n-1}\right) \\
& \leq 2 h^{-\alpha} g_{0}^{(\alpha)}\left\|\Lambda_{h}^{\alpha} u^{n-1}\right\|^{2} \\
& \leq 8 h^{-\alpha} g_{0}^{(\alpha)} \varepsilon^{0} .
\end{aligned}
$$

Noticing that

$$
\left(\delta_{h}^{\alpha} \omega, \omega\right)=\left(\Lambda_{h}^{\alpha} \omega, \Lambda_{h}^{\alpha} \omega\right) \geq 0
$$

and

$$
\left(\frac{\cos u^{n-1}-\cos \left(\omega+u^{n-1}\right)}{\omega}, \omega\right)=h \sum_{i=1}^{M-1}\left(\cos u_{i}^{n-1}-\cos \left(\omega_{i}+u_{i}^{n-1}\right)\right) \leq 2 L
$$

where $L=X_{R}-X_{L}$, we have

$$
(\digamma(\omega), \omega) \geq \frac{1}{4} \frac{\|\omega\|^{2}}{\tau^{2}}-\left[\left(8+4 \tau^{2} h^{-\alpha} g_{0}^{(\alpha)}\right) \varepsilon^{0}+2 L\right] .
$$

Let

$$
K=2 \tau \sqrt{\left(8+4 \tau^{2} h^{-\alpha} g_{0}^{(\alpha)}\right) \varepsilon^{0}+2 L} .
$$

Then, for any $\|\omega\|>K$, we have $(\digamma(\omega), \omega)>0$. By Lemma 3.6 there exists $\omega \in X$ such that $\digamma(\omega)=0$. Setting $\omega=u^{n+1}-u^{n-1}$, we get the desired result.

Theorem 3.4 The solution of the difference scheme (2.9)-(2.11) is unique. 
Proof Suppose that two sequences $u^{0}, u^{1}, \ldots, u^{n}, v$ and $u^{0}, u^{1}, \ldots, u^{n}, v^{\prime}$ both satisfy the difference scheme (2.9)-(2.11). Let $v^{*}=v-v^{\prime}$. Then we obtain that

$$
\frac{1}{\tau^{2}} v_{i}^{*}+\delta_{h}^{\alpha} v_{i}^{*}-p_{i}=0, \quad 1 \leq i \leq M-1,
$$

where $\left\{p_{i}\right\}$ are given by (3.8).

Making the inner product of (3.6) with $v^{*}$, we obtain that

$$
\left(\frac{1}{\tau^{2}} v^{*}, v^{*}\right)+\left(\delta_{h}^{\alpha} v^{*}, v^{*}\right)-\left(p, v^{*}\right)=0
$$

Noticing that

$$
\begin{aligned}
p_{i} & =\frac{\cos v_{i}-\cos u_{i}^{n-1}}{v_{i}-u_{i}^{n-1}}-\frac{\cos v_{i}^{\prime}-\cos u_{i}^{n-1}}{v_{i}^{\prime}-u_{i}^{n-1}} \\
& =\int_{0}^{1} \sin \left[\lambda v_{i}^{\prime}+(1-\lambda) u_{i}^{n-1}\right] d \lambda-\int_{0}^{1} \sin \left[\lambda v_{i}+(1-\lambda) u_{i}^{n-1}\right] d \lambda \\
& =-2 \int_{0}^{1} \cos \left[\lambda \frac{v_{i}+v_{i}^{\prime}}{2}+(1-\lambda) u_{i}^{n-1}\right] \sin \left(\lambda \frac{v_{i}^{*}}{2}\right) d \lambda
\end{aligned}
$$

we have

$$
\left|p_{i} v_{i}^{*}\right| \leq \frac{1}{2}\left(v_{i}^{*}\right)^{2}
$$

and therefore

$$
\left|\left(p, v^{*}\right)\right| \leq \frac{1}{2}\left\|v^{*}\right\|^{2}
$$

Thus for the right-hand side of (3.7), we get

$$
\left(\frac{1}{\tau^{2}} v^{*}, v^{*}\right)+\left(\delta_{h}^{\alpha} v^{*}, v^{*}\right)-\left(p, v^{*}\right) \geq\left(\frac{1}{\tau^{2}}-\frac{1}{2}\right)\left\|v^{*}\right\|^{2} .
$$

Then supposing that $\tau<\sqrt{2}$ and combining (3.7) and (3.9), we derive

$$
\left\|v^{*}\right\|=0, \quad \text { i.e., } v=v^{\prime} .
$$

The proof ends.

\section{Denote}

$$
e_{i}^{n}=U_{i}^{n}-u_{i}^{n}, \quad 0 \leq i \leq M, 0 \leq n \leq N .
$$

Theorem 3.5 Assume that problem (1.2)-(1.4) has a smooth solution and $\left\{u_{i}^{n} \mid 0 \leq i \leq\right.$ $M, 0 \leq n \leq N\}$ is the solution of the finite difference scheme (2.9)-(2.11). If $\frac{\tau^{2}}{h^{\alpha}} \leq S(0<$ $S<+\infty)$, then there exists a positive constant $C$ such that

$$
\left\|e^{n}\right\|_{l_{h}^{\infty}} \leq C\left(h^{2}+\tau^{2}\right)
$$


Proof Subtracting (2.9)-(2.11) from (2.6)-(2.8), respectively, we get the following error equations:

$$
\begin{aligned}
& \left(e_{i}^{n}\right)_{t \bar{t}}+\delta_{h}^{\alpha} \bar{e}_{i}^{n}=V\left(\bar{e}_{i}^{n}\right)+r_{i}^{n}, \quad 1 \leq i \leq M-1,1 \leq n \leq N, \\
& e_{0}^{n}=0, \quad e_{M}^{n}=0, \quad 0 \leq n \leq N, \\
& e_{i}^{0}=0, \quad 1 \leq i \leq M-1,
\end{aligned}
$$

where

$$
\begin{aligned}
V\left(\bar{e}_{i}^{n}\right)= & \zeta\left(\bar{U}_{i}^{n}\right)-\zeta\left(\bar{u}_{i}^{n}\right) \\
= & -2 \int_{0}^{1} \cos \left[\lambda \frac{U_{i}^{n+1}+u_{i}^{n+1}}{2}+(1-\lambda) \frac{U_{i}^{n-1}+u_{i}^{n-1}}{2}\right] \\
& \times \sin \left[\lambda \frac{U_{i}^{n+1}-u_{i}^{n+1}}{2}+(1-\lambda) \frac{U_{i}^{n-1}-u_{i}^{n-1}}{2}\right] d \lambda .
\end{aligned}
$$

Similarly to the proof of Theorem 3.1, making the discrete inner product of (3.10) with $2 e_{\hat{t}}^{n}$, we get that

$$
\begin{aligned}
& \left(\left\|e_{t}^{n}\right\|^{2}\right)_{\bar{t}}+\frac{1}{2 \tau}\left[\left(\left\|\Lambda_{h}^{\alpha} e^{n+1}\right\|^{2}+\left\|\Lambda_{h}^{\alpha} e^{n}\right\|^{2}\right)-\left(\left\|\Lambda_{h}^{\alpha} e^{n}\right\|^{2}+\left\|\Lambda_{h}^{\alpha} e^{n-1}\right\|^{2}\right)\right] \\
& =\left(V\left(\bar{e}^{n}\right)+r^{n}, 2 e_{\hat{t}}^{n}\right) .
\end{aligned}
$$

By summing this equality for $n=1,2, \ldots, k(k \tau \leq T)$, we derive

$$
\begin{aligned}
& \left\|e_{t}^{k}\right\|^{2}+\frac{1}{2}\left\|\Lambda_{h}^{\alpha} e^{k+1}\right\|^{2}+\frac{1}{2}\left\|\Lambda_{h}^{\alpha} e^{k}\right\|^{2} \\
& =\left\|e_{t}^{0}\right\|^{2}+\frac{1}{2}\left\|\Lambda_{h}^{\alpha} e^{1}\right\|^{2}+\frac{1}{2}\left\|\Lambda_{h}^{\alpha} e^{0}\right\|^{2}+\tau \sum_{n=1}^{k}\left(V\left(\bar{e}^{n}\right)+r^{n}, 2 e_{\hat{t}}^{n}\right) .
\end{aligned}
$$

From (3.13) we get

$$
\begin{aligned}
\left|V\left(\bar{e}_{i}^{n}\right)\right| & \leq\left|2 \int_{0}^{1} \sin \left[\lambda \frac{e_{i}^{n+1}}{2}+(1-\lambda) \frac{e_{i}^{n-1}}{2}\right] d \lambda\right| \\
& \leq \int_{0}^{1}\left|\lambda e_{i}^{n+1}+(1-\lambda) e_{i}^{n-1}\right| d \lambda \\
& \leq \int_{0}^{1}\left|\tau \lambda\left(e_{i}^{n}\right)_{t}+\lambda e_{i}^{n}+(1-\lambda) e_{i}^{n-1}\right| d \lambda \\
& =\frac{1}{2} \tau\left|\left(e_{i}^{n}\right)_{t}\right|+\frac{1}{2}\left|e_{i}^{n}\right|+\frac{1}{2}\left|e_{i}^{n-1}\right|,
\end{aligned}
$$

and hence

$$
\left\|V\left(\bar{e}^{n}\right)\right\|^{2} \leq \frac{3}{4}\left(\tau^{2}\left\|e_{t}^{n}\right\|^{2}+\left\|e^{n}\right\|^{2}+\left\|e^{n-1}\right\|^{2}\right) .
$$


Noticing that $e_{i}^{n}=e_{i}^{0}+\tau \sum_{j=0}^{n-1}\left(e_{i}^{j}\right)_{t}$ and using the Cauchy-Schwarz inequality, we obtain

$$
\begin{aligned}
\left\|e^{n}\right\|^{2} & \leq 2\left\|e^{0}\right\|^{2}+2 \tau^{2}\left\|\sum_{j=0}^{n-1} e_{t}^{j}\right\|^{2} \\
& \leq 2\left\|e^{0}\right\|^{2}+2 T \tau \sum_{j=0}^{n-1}\left\|e_{t}^{j}\right\|^{2} .
\end{aligned}
$$

According to (3.16)-(3.17) and noticing that $e_{\hat{t}}^{n}=\frac{1}{2} e_{t}^{n}+\frac{1}{2} e_{t}^{n-1}$, we get

$$
\begin{aligned}
\left|\tau \sum_{n=1}^{k}\left(V\left(\bar{e}^{n}\right), 2 e_{\hat{t}}^{n}\right)\right| \leq & \tau \sum_{n=1}^{k}\left(\left\|V\left(\bar{e}^{n}\right)\right\|^{2}+\frac{1}{2}\left\|e_{t}^{n}\right\|^{2}+\frac{1}{2}\left\|e_{t}^{n-1}\right\|^{2}\right) \\
\leq & 3 T\left\|e^{0}\right\|^{2}+\frac{3 \tau^{3}+2 \tau}{4}\left\|e_{t}^{k}\right\|^{2} \\
& +\left(\frac{3}{4} \tau^{3}+3 T^{2} \tau+\tau\right) \sum_{n=0}^{k-1}\left\|e_{t}^{n}\right\|^{2} .
\end{aligned}
$$

On the other hand,

$$
\begin{aligned}
\left|\tau \sum_{n=1}^{k}\left(r^{n}, 2 e_{\hat{t}}^{n}\right)\right| & \leq \tau \sum_{n=1}^{k}\left(\left\|r^{n}\right\|^{2}+\left\|e_{\hat{t}}^{n}\right\|^{2}\right) \\
& \leq \tau \sum_{n=1}^{k}\left\|r^{n}\right\|^{2}+\frac{1}{2} \tau \sum_{n=0}^{k}\left\|e_{t}^{n}\right\|^{2}+\frac{1}{2} \tau \sum_{n=0}^{k-1}\left\|e_{t}^{n}\right\|^{2} .
\end{aligned}
$$

Substituting (3.18) and (3.19) into (3.15) and noticing that $e^{0}=0$, we get

$$
\begin{gathered}
\left(1-\left(\frac{3 \tau^{3}}{4}+\tau\right)\right)\left\|e_{t}^{k}\right\|^{2}+\frac{1}{2}\left\|\Lambda_{h}^{\alpha} e^{k+1}\right\|^{2}+\frac{1}{2}\left\|\Lambda_{h}^{\alpha} e^{k}\right\|^{2} \\
\leq\left\|e_{t}^{0}\right\|^{2}+\frac{1}{2}\left\|\Lambda_{h}^{\alpha} e^{1}\right\|^{2}+\tau \sum_{n=1}^{k}\left\|r^{n}\right\|^{2} \\
+\left(\frac{3}{4} \tau^{3}+3 T^{2} \tau+2 \tau\right) \sum_{n=0}^{k-1}\left\|e_{t}^{n}\right\|^{2}
\end{gathered}
$$

In addition,

$$
\begin{gathered}
\left(1-\left(\frac{3 \tau^{3}}{4}+\tau\right)\right)\left\|e_{t}^{k}\right\|^{2}+\frac{1}{2}\left\|\Lambda_{h}^{\alpha} e^{k+1}\right\|^{2}+\frac{1}{2}\left\|\Lambda_{h}^{\alpha} e^{k}\right\|^{2} \\
\geq\left(1-\left(\frac{3 \tau^{3}}{4}+\tau\right)\right)\left\|e_{t}^{k}\right\|^{2}+\frac{1}{2} \tau^{2}\left\|\Lambda_{h}^{\alpha} e_{t}^{k}\right\|^{2} \\
\geq\left(1-\left(\frac{3 \tau^{3}}{4}+\tau\right)+\frac{1}{2} \tau^{2} h^{-\alpha} \lambda_{\min }(G)\right)\left\|e_{t}^{k}\right\|^{2}
\end{gathered}
$$


Supposing that $\sigma=1-\left(\frac{3 \tau^{3}}{4}+\tau\right)+\frac{1}{2} \tau^{2} h^{-\alpha} \lambda_{\min }(G)>0$ (it is easily to verify that when $\tau \leq$ 0.72 , the condition is satisfied), then inequality (3.20) can be rewritten as

$$
\begin{aligned}
\left\|e_{t}^{k}\right\|^{2}+\frac{1}{2 \sigma}\left\|\Lambda_{h}^{\alpha} e^{k}\right\|^{2} \leq & \frac{1}{\sigma}\left\|e_{t}^{0}\right\|^{2}+\frac{1}{2 \sigma}\left\|\Lambda_{h}^{\alpha} e^{1}\right\|^{2}+\frac{\tau}{\sigma} \sum_{n=1}^{k}\left\|r^{n}\right\|^{2} \\
& +\frac{3 \tau^{2}+12 T^{2}+8}{4 \sigma} \tau \sum_{n=0}^{k-1}\left(\left\|e_{t}^{n}\right\|^{2}+\frac{1}{2 \sigma}\left\|\Lambda_{h}^{\alpha} e^{n}\right\|^{2}\right) .
\end{aligned}
$$

Let

$$
\begin{aligned}
& \rho(k)=\frac{1}{\sigma}\left\|e_{t}^{0}\right\|^{2}+\frac{1}{2 \sigma}\left\|\Lambda_{h}^{\alpha} e^{1}\right\|^{2}+\frac{\tau}{\sigma} \sum_{n=1}^{k}\left\|r^{n}\right\|^{2}, \\
& H(k)=\left\|e_{t}^{k}\right\|^{2}+\frac{1}{2 \sigma}\left\|\Lambda_{h}^{\alpha} e^{k}\right\|^{2} .
\end{aligned}
$$

Substituting (3.22) into (3.21), we derive

$$
H(k) \leq \rho(k)+\frac{3 \tau^{2}+12 T^{2}+8}{4 \sigma} \tau \sum_{n=0}^{k-1} H(n) .
$$

Applying Lemma 3.3 to $\rho(k)$ and $H(k)$, we arrive at

$$
H(k) \leq \rho(k) e^{\frac{3 \tau^{2}+12 T^{2}+8}{4 \sigma} k \tau} \leq \rho(k) e^{\frac{\left(3 \tau^{2}+12 T^{2}+8\right) T}{4 \sigma}} .
$$

Noticing that if $\tau$ and $h$ are sufficiently small, then we have

$$
e_{i}^{0}=0, \quad r_{i}^{n}=O\left(h^{2}+\tau^{2}\right), \quad 1 \leq i \leq M-1,1 \leq n \leq N-1 .
$$

Applying the Taylor expansion, we get

$$
u_{i}^{1}=u_{i}^{0}+\tau \psi\left(x_{i}\right)+\frac{\tau^{2}}{2}\left[-(-\Delta)^{\frac{\alpha}{2}} u_{i}^{0}-\sin \left(u_{i}^{0}\right)\right]+O\left(\tau^{3}\right), \quad 1 \leq i \leq M-1 .
$$

According to Lemma 2.1, we obtain

$$
-(-\Delta)^{\frac{\alpha}{2}} u_{i}^{0}=-\delta_{h}^{\alpha} u_{i}^{0}+O\left(h^{2}\right) .
$$

Therefore, we have the following equality:

$$
e_{i}^{1}=O\left(\tau^{3}+h^{2} \tau^{2}\right), \quad 1 \leq i \leq M-1 .
$$

Due to $e_{i}^{0}=0, \frac{\tau^{2}}{h^{\alpha}}<S$, and (3.24), we derive

$$
\begin{aligned}
& \left\|e_{t}^{0}\right\|^{2} \leq L \max _{1 \leq i \leq M-1}\left(\frac{e_{i}^{1}}{\tau}\right)^{2} \leq C\left(\tau^{2}+h^{2} \tau\right)^{2} \leq C\left(\tau^{2}+h^{2}\right)^{2}=O\left(\tau^{2}+h^{2}\right)^{2}, \\
& \left\|\Lambda_{h}^{\alpha} e^{1}\right\|^{2} \leq h^{-\alpha} \lambda_{\max }(G)\left\|e^{1}\right\|^{2}
\end{aligned}
$$




$$
\begin{aligned}
& \leq 2 h^{-\alpha} g_{0}^{(\alpha)} L \max _{1 \leq i \leq M-1}\left(e_{i}^{1}\right)^{2} \\
& \leq C \frac{\tau^{2}}{h^{\alpha}}\left(\tau^{2}+h^{2} \tau\right)^{2} \\
& \leq C \cdot S\left(\tau^{2}+h^{2}\right)^{2} \\
& =O\left(\tau^{2}+h^{2}\right)^{2} .
\end{aligned}
$$

On the other hand,

$$
\tau \sum_{n=1}^{k}\left\|r^{n}\right\|^{2} \leq T \max _{1 \leq n \leq k}\left\|r^{n}\right\|^{2} \leq T \cdot L \max _{1 \leq n \leq k, 1 \leq i \leq M-1}\left(r_{i}^{n}\right)^{2}=O\left(\tau^{2}+h^{2}\right)^{2} .
$$

Combining (3.25), (3.26), and (3.27), we derive

$$
\rho(k)=O\left(h^{2}+\tau^{2}\right)^{2}
$$

Furthermore, from (3.23) and (3.28) we immediately obtain the following results:

$$
\left\|e_{t}^{k}\right\| \leq C\left(h^{2}+\tau^{2}\right), \quad\left\|\Lambda_{h}^{\alpha} e^{k}\right\| \leq C\left(h^{2}+\tau^{2}\right) .
$$

Hence

$$
\frac{\left\|e^{k+1}\right\|-\left\|e^{k}\right\|}{\tau} \leq\left\|e_{t}^{k}\right\| \leq C\left(h^{2}+\tau^{2}\right) .
$$

By summing this equality for $k=0,1, \ldots, n-1(n \tau \leq T)$, we get

$$
\left\|e^{n}\right\| \leq n \tau C\left(h^{2}+\tau^{2}\right)+\left\|e^{0}\right\|=O\left(h^{2}+\tau^{2}\right) .
$$

It follows from Lemma 3.1 and Lemma 3.5 that

$$
\left|e^{n}\right|_{H^{\alpha / 2}}^{2} \leq\left(\frac{\pi}{2}\right)^{\alpha}\left\|\Lambda_{h}^{\alpha} e^{n}\right\|^{2}=O\left(h^{2}+\tau^{2}\right)^{2}
$$

and hence

$$
\left\|e^{n}\right\|_{H^{\alpha / 2}}^{2}=\left\|e^{n}\right\|^{2}+\left|e^{n}\right|_{H^{\alpha / 2}}^{2}=O\left(h^{2}+\tau^{2}\right)^{2} .
$$

According to Lemma 3.4,

$$
\left\|e^{n}\right\|_{l_{h}^{\infty}} \leq C\left\|e^{n}\right\|_{H^{\alpha / 2}}=O\left(h^{2}+\tau^{2}\right) .
$$

Thus we get the desired results.

\section{The implementation issue and feasibility analysis of the numerical scheme}

\subsection{A revised Newton method for the proposed difference scheme}

In this subsection, we mainly introduce how to implement the difference scheme (2.9)(2.11). First, we rewrite the difference scheme as follows:

$$
\left(\frac{1}{\tau^{2}} I+\frac{1}{2 h^{\alpha}} G\right) u^{n+1}-Q\left(u^{n+1}, u^{n-1}\right)+g=0 \quad(n=1,2, \ldots, N-1),
$$


where

$$
\begin{aligned}
& Q\left(u^{n+1}, u^{n-1}\right)=\left(\zeta\left(\bar{u}_{1}^{n}\right), \zeta\left(\bar{u}_{2}^{n}\right), \ldots, \zeta\left(\bar{u}_{M-1}^{n}\right)\right)^{T}, \\
& u^{n}=\left(u_{1}^{n}, u_{2}^{n}, \ldots, u_{M-1}^{n}\right)^{T}, \\
& g=\frac{1}{2 h^{\alpha}} G u^{n-1}-\frac{1}{\tau^{2}}\left(2 u^{n}-u^{n-1}\right) .
\end{aligned}
$$

Note that, at each time level $k=n+1, u^{n}, u^{n-1}$, and $g$ are constants. If we define

$$
F(x)=\left(\frac{1}{\tau^{2}} I+\frac{1}{2 h^{\alpha}} G\right) x-Q\left(x, u^{n-1}\right)+g \quad\left(x \in R^{M-1}\right),
$$

then $u^{n+1}$ turns out to be a solution of the nonlinear equation

$$
F(x)=0 .
$$

In addition, letting $u^{n+1}=x$, any solution of the nonlinear equation (4.2) satisfies the difference equations (2.9). Hence the solution of (4.2) is unique.

Applying the Newton method to solve system (4.2), we get

$$
J\left(x^{s}\right)\left(x^{s+1}-x^{s}\right)=-F\left(x^{s}\right), \quad s=0,1,2, \ldots,
$$

that is,

$$
J\left(u^{n+1(s)}\right)\left(u^{n+1(s+1)}-u^{n+1(s)}\right)=-F\left(u^{n+1(s)}\right),
$$

where

$$
J\left(u^{n+1(s)}\right)=\frac{1}{\tau^{2}} I+\frac{1}{2 h^{\alpha}} G-\operatorname{diag}\left(q_{1}^{n+1}, q_{2}^{n+1}, \ldots, q_{M-1}^{n+1}\right)
$$

and

$$
q_{i}^{n+1}=\left.\frac{d}{d x_{i}} Q\left(x_{i}, u_{i}^{n-1}\right)\right|_{x_{i}=u_{i}^{n+1(s)}}= \begin{cases}-\cos \left(u_{i}^{n+1(s)}\right) & \text { if } u_{i}^{n+1(s)}=u_{i}^{n-1} \\ \Theta\left(u_{i}^{n+1(s)}, u_{i}^{n-1}\right) & \text { if } u_{i}^{n+1(s)} \neq u_{i}^{n-1}\end{cases}
$$

with

$$
\begin{aligned}
\left|\Theta\left(u_{i}^{n+1(s)}, u_{i}^{n-1}\right)\right| & =\left|\left[\frac{d}{d x_{i}} \int_{0}^{1} \sin \left(\lambda x_{i}+(1-\lambda) u_{i}^{n-1}\right) d \lambda\right]_{x_{i}=u_{i}^{n+1(s)}}\right| \\
& =\left|\int_{0}^{1} \lambda \cos \left(\lambda u_{i}^{n+1(s)}+(1-\lambda) u_{i}^{n-1}\right) d \lambda\right| \\
& \leq \int_{0}^{1} \lambda d \lambda \\
& =\frac{1}{2} .
\end{aligned}
$$


Therefore $\left|q_{i}^{n+1}\right| \leq 1(i=1,2, \ldots, M-1)$. Hence, as $\tau \rightarrow 0$ or $h \rightarrow 0$, we have

$$
\frac{\left|q_{i}^{n+1}\right|}{\frac{1}{\tau^{2}}+\frac{1}{2 h^{\alpha}} g_{0}^{(\alpha)}} \rightarrow 0, \quad i=1,2, \ldots, M-1
$$

If we apply the Newton method to solve system (4.2), then we have to renew the Jacobian matrix of $F$ at each stage, which will be very expensive. However, from (4.4) we observe that the contribution of the term $Q\left(x, u^{n-1}\right)$ to the Jacobian matrix can be omitted when $\tau$ and $h$ are sufficiently small. So we revise the Jacobian matrix as

$$
J=\frac{1}{\tau^{2}} I+\frac{1}{2 h^{\alpha}} G
$$

Then the revised Newton method for solving equations (4.2) is given as follows:

$$
J\left(x^{s+1}-x^{s}\right)=-F\left(x^{s}\right), \quad s=0,1,2, \ldots
$$

\subsection{Feasibility analysis}

Now we consider the feasibility of the revised Newton method for solving equations (4.2).

Denote

$$
A=J\left(x^{s}\right), \quad y=\left(x^{s+1}-x^{s}\right), \quad b=-F\left(x^{s}\right) .
$$

Then equations (4.3) change into $A y=b$. Using the revised Newton method for equations (4.2), we can rewrite the corresponding perturbed equations as

$$
(A+\delta A)(y+\delta y)=b,
$$

where

$$
A+\delta A=J, \quad \delta A=-\operatorname{diag}\left(q_{1}^{n+1}, q_{2}^{n+1}, \ldots, q_{M-1}^{n+1}\right)
$$

Applying the classic perturbation analysis theory, we get

$$
\frac{\|\delta y\|}{\|y\|} \leq \frac{\operatorname{cond}(A) \frac{\|\delta A\|}{\|A\|}}{1-\operatorname{cond}(A) \frac{\|\delta A\|}{\|A\|}}
$$

where

$$
\begin{aligned}
& \|y\|^{2}=y^{T} y, \quad\|A\|=\max \left\{\sqrt{\lambda}: \lambda \text { is an eigenvalue of } A^{T} A\right\}, \\
& \operatorname{cond}(A)=\left\|A^{-1}\right\|\|A\|=\frac{\lambda_{\text {max }}(A)}{\lambda_{\min }(A)} .
\end{aligned}
$$

According to (4.4), as $\tau, h \rightarrow 0$, we have $\frac{\|\delta A\|}{\|A\|} \rightarrow 0$ and

$$
\operatorname{cond}(A) \leq \frac{\frac{1}{\tau^{2}}+\frac{1}{h^{\alpha}} g_{0}^{(\alpha)}+\max \left\{q_{i}^{n+1}: i=1,2, \ldots, M-1\right\}}{\frac{1}{\tau^{2}}+\min \left\{q_{i}^{n+1}: i=1,2, \ldots, M-1\right\}} \approx 1+\frac{\tau^{2}}{h^{\alpha}} g_{0}^{(\alpha)}
$$


Table 1 The convergence order in spatial direction with $\alpha=1.75,1.95, \tau=1 / 256$ at time $t=1$

\begin{tabular}{llllr}
\hline $\mathrm{h}$ & $E(h)(\alpha=1.75)$ & order & $E(h)(\alpha=1.95)$ & order \\
\hline 1 & 0.071175803333281 & - & 0.083949535804123 & \\
$1 / 2$ & 0.018280687400889 & 1.9611 & 0.020521577513857 & 2.0324 \\
$1 / 4$ & 0.004463589467647 & 2.0340 & 0.004906614930621 & 2.0643 \\
$1 / 8$ & 0.001108210787441 & 2.0100 & 0.001213495898485 & 2.0156 \\
\hline
\end{tabular}

Hence, if $\tau, h \rightarrow 0$ and $\frac{\tau^{2}}{h^{\alpha}}<\infty$, we have

$$
\frac{\|\delta y\|}{\|y\|} \rightarrow 0
$$

In summary, when $h, \tau$ are sufficiently small, the revised Newton method for solving equations (4.2) is feasible.

\section{Numerical results}

In this section, we present a few numerical results to verify the effectiveness of the conservative difference scheme (2.9)-(2.11) and the efficiency of the revised Newton method developed in Sect. 4 for implementation of the difference scheme. All numerical computations were carried out using MATLAB on a DELL OptiPlex 3046 computer Intel(R) Core(TM) I5-6500, 3.2 GHz and 4 GB RAM.

Example 1 We take the following initial conditions:

$$
\begin{cases}u(x, 0)=0, & x \in\left[x_{L}, x_{R}\right], \\ u_{t}(x, 0)=4 \operatorname{sech} x, & x \in\left[x_{L}, x_{R}\right] .\end{cases}
$$

Firstly, we test the convergence order of the difference scheme (2.9)-(2.11). Denote

$$
\begin{aligned}
& E(h)=\max _{0 \leq i \leq M}\left|u_{i}^{n}(\tau, h)-u_{2 i}^{n}(\tau, h / 2)\right|, \\
& E(\tau)=\max _{0 \leq i \leq M}\left|u_{i}^{n}(\tau, h)-u_{i}^{2 n}(\tau / 2, h)\right|, \\
& p=\log _{2}[E(h) / E(h / 2)], \\
& q=\log _{2}[E(\tau) / E(\tau / 2)],
\end{aligned}
$$

where $p, q$ denote the space convergence order and time convergence order, respectively. We apply the difference scheme (2.9)-(2.11) with fixed time step $\tau=1 / 256$ and different space steps to solve the problem with different values of fractional order $\alpha$ with $-x_{L}=x_{R}=$ 40 and $T=1$. The computation results are presented in Table 1 . Also with a fixed space step $h=1 / 128$, we use different time steps to solve these problems. The computation results are listed in Table 2. According to Tables 1 and 2, the convergence orders in space and time are both consistent with theoretical results. Moreover, we depict the numerical solution of the problem with $\alpha=1.75, h=1 / 16$, and $\tau=1 / 200$ in Fig. 1 , which shows that the results we get are qualitatively similar to those obtained in $[4,20]$.

Secondly, we pay attention to investigate the relationship between the fractional order $\alpha$ and the shape of the soliton for the problem with different fractional order $\alpha$. We choose 
Table 2 The convergence order in temporal direction with $\alpha=1.75,1.95, h=1 / 128$ at time $t=1$

\begin{tabular}{lllll}
\hline$\tau$ & $E(\tau)(\alpha=1.75)$ & order & $E(\tau)(\alpha=1.95)$ & order \\
\hline $1 / 4$ & 0.076360499008544 & - & 0.077602598777544 & - \\
$1 / 8$ & 0.020013445313199 & 1.9319 & 0.020188901056871 & 1.9425 \\
$1 / 16$ & 0.005060174214254 & 1.9837 & 0.005092254511141 & 1.9872 \\
$1 / 32$ & 0.001268571271052 & 1.9960 & 0.001275824597592 & 1.9969 \\
\hline
\end{tabular}

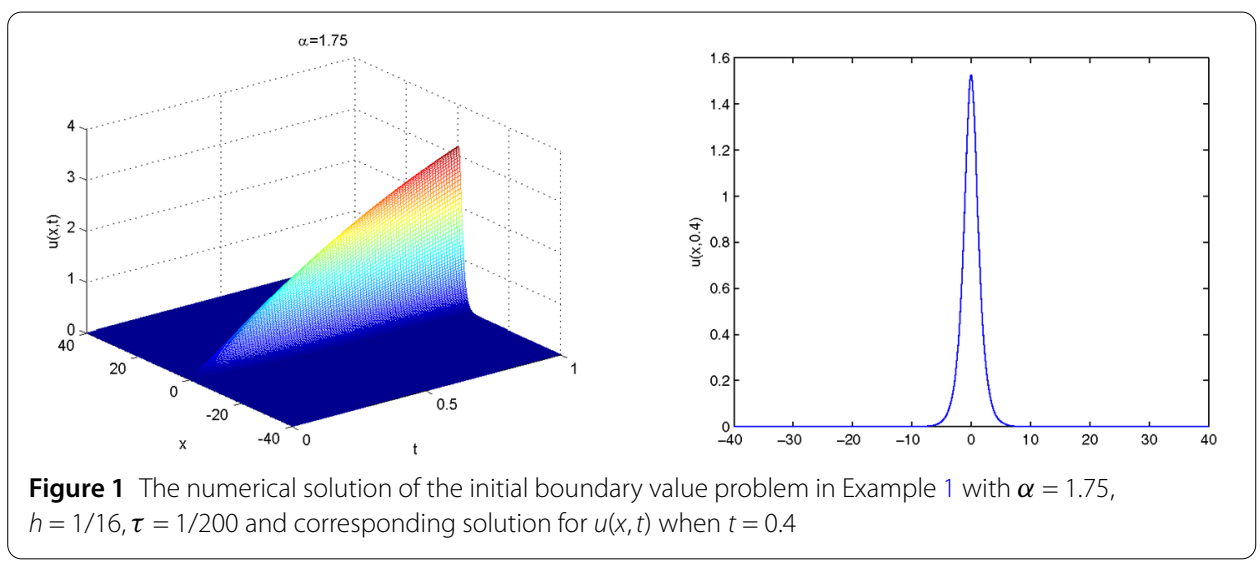

$-x_{L}=x_{R}=100$ and $T=150$ for the problem. The numerical results are presented in Fig. 2, from which we derive that the shape of the soliton is changes dramatically when value of the order $\alpha$ is changed from 2.0 to 1.99 . When $1<\alpha<2$, along with $\alpha$ becoming smaller, the period of the soliton turns out to be smaller.

Finally, we test the discrete energy conservation law of the difference scheme (2.9)(2.11). The values of the discrete energy at different moments for $\alpha=1.1,1.75,1.99,2.0$ are listed in Table 3, where the numerical results are derived with $h=\tau=\frac{1}{10}$. From Table 3 we obtain that the difference scheme preserves the energy conservation very well and the energy varies with different $\alpha$.

Example 2 We take the following initial conditions:

$$
\begin{cases}u(x, 0)=3.2 \operatorname{sech} x, & x \in\left[x_{L}, x_{R}\right], \\ u_{t}(x, 0)=0, & x \in\left[x_{L}, x_{R}\right] .\end{cases}
$$

Firstly, the convergence order of the difference scheme (2.9)-(2.11) in space and time are tested for the problem with different values of fractional order $\alpha$ with $-x_{L}=x_{R}=40$ and $T=1$. The computation results are presented in Tables 4 and 5 . According to Tables 4 and 5 , the convergence orders in space and time are both consistent with theoretical results.

Secondly, we test the discrete energy conservation law of the difference scheme (2.9)-(2.11) for the problem with different values of fractional order $\alpha$ with $-x_{L}=$ $x_{R}=100$ and $T=100$. The values of the discrete energy at different moments for $\alpha=1.1,1.5,1.95,2.0$ are listed in Table 6 , where the numerical results are derived with $h=\frac{1}{10}$ and $\tau=\frac{1}{20}$. From Table 6 we can observe that the difference scheme can preserve the energy conservation very well in a long time interval and the energy depends on the order $\alpha$. Moreover, the numerical results are presented in Fig. 3. We find that 


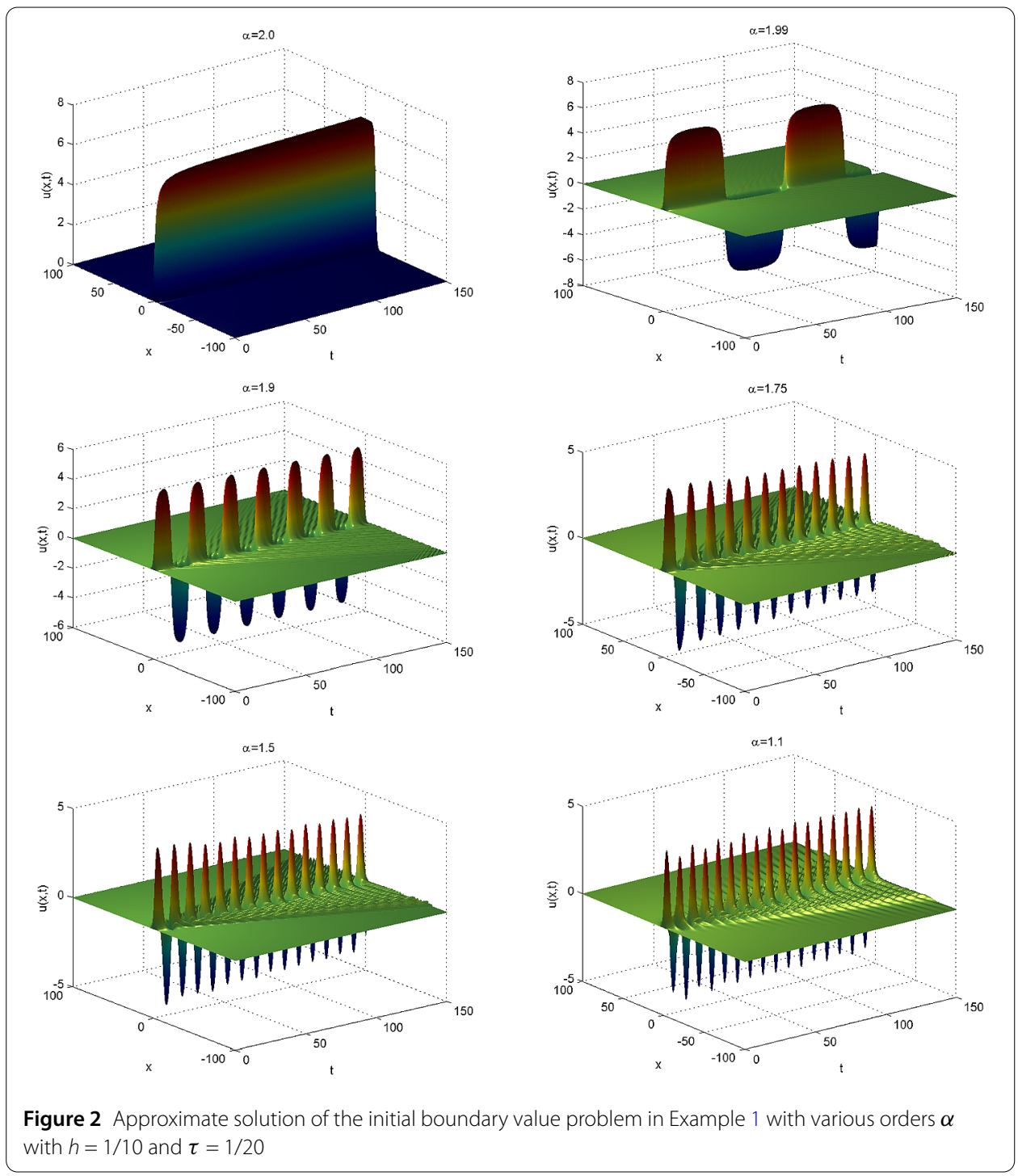

the fractional order $\alpha$ affects the shape of the wave of the initial boundary problem.

Finally, we test the efficiency of the revised Newton method developed in Sect. 4. In Table 7, we display the consumed CPU time to implement the difference scheme by the regular Newton method and the revised Newton method for different space steps for the problem in Example 2 with $\alpha=1.75,-x_{L}=x_{R}=40, T=1$, and $\tau=1 / 200$. We conclude that the revised Newton method significantly reduces the computational cost of the difference scheme. It reduces the CPU time from more than 2 hours 12 minutes consumed by the regular Newton method to less than 8 minutes for the model with space step $h=\frac{1}{128}$.

\section{Conclusions}

In this paper, we propose a conservative implicit difference scheme for SGEs with the Riesz space fractional derivative. We give a rigorous theoretical analysis of its conservation property, boundedness, and convergence. We introduce a revised Newton iterative 
Table $3 \varepsilon^{n}$ at $t=t_{n}$ for different values of $\alpha$ with $h=\frac{1}{10}, \tau=\frac{1}{20}$

\begin{tabular}{lllll}
\hline $\mathrm{t}$ & $\alpha=1.1$ & $\alpha=1.75$ & $\alpha=1.99$ & $\alpha=2.0$ \\
\hline 0 & 16.028375166427221 & 16.026853085312613 & 16.026621262922639 & 16.026614500015587 \\
10 & 16.028375167453508 & 16.026853083065745 & 16.026621263595434 & 16.026614500665112 \\
20 & 16.028375167026866 & 16.026853083341813 & 16.026621263623838 & 16.026614500693057 \\
30 & 16.028375169429935 & 16.026853082109128 & 16.026621263611123 & 16.026614500697043 \\
40 & 16.028375169276917 & 16.026853082184989 & 16.026621262942438 & 16.026614500697779 \\
50 & 16.028375174037464 & 16.026853082023688 & 16.026621263929581 & 16.026614500697725 \\
60 & 16.028375172694766 & 16.026853081374647 & 16.026621263991700 & 16.026614500697306 \\
70 & 16.028375173733984 & 16.026853080731076 & 16.026621263988194 & 16.026614500697072 \\
80 & 16.028375174760189 & 16.026853081190453 & 16.026621263850092 & 16.026614500696731 \\
90 & 16.028375175956295 & 16.026853082788968 & 16.026621264363811 & 16.026614500696208 \\
100 & 16.028375178909357 & 16.026853082989092 & 16.026621264523360 & 16.026614500696372 \\
110 & 16.028375178244517 & 16.026853083445701 & 16.026621264528124 & 16.026614500695700 \\
120 & 16.028375179698422 & 16.026853085066683 & 16.026621264471139 & 16.026614500695260 \\
130 & 16.028375180814812 & 16.026853085383230 & 16.026621264061959 & 16.026614500694873 \\
140 & 16.028375181940007 & 16.026853087126149 & 16.026621264895454 & 16.026614500694507 \\
$150-\tau$ & 16.028375183321515 & 16.026853087643513 & 16.026621264907444 & 16.026614500694265 \\
\hline
\end{tabular}

Table 4 The convergence order in spatial direction with $\alpha=1.5,1.95, \tau=1 / 400$ at time $t=1$

\begin{tabular}{lllll}
\hline $\mathrm{h}$ & $E(h)(\alpha=1.5)$ & order & $E(h)(\alpha=1.95)$ & order \\
\hline 1 & 0.175728570098761 & - & 0.130474954167071 & - \\
$1 / 2$ & 0.0309275588960760 & 2.0768 & 0.033480284841195 & 2.3920 \\
$1 / 4$ & 0.007465839195053 & 2.0505 & 0.007679396860666 & 2.1242 \\
$1 / 8$ & 0.002086595144613 & 1.8392 & 0.001888044160022 & 2.0241 \\
\hline
\end{tabular}

Table 5 The convergence order in temporal direction with $\alpha=1.5,1.95, h=1 / 300$ at time $t=1$

\begin{tabular}{lllll}
\hline$\tau$ & $E(\tau)(\alpha=1.5)$ & order & $E(\tau)(\alpha=1.95)$ & order \\
\hline $1 / 20$ & $8.0262 \mathrm{e}-004$ & - & $6.1903 \mathrm{e}-004$ & - \\
$1 / 40$ & $2.0469 \mathrm{e}-004$ & 1.9713 & $1.6227 \mathrm{e}-004$ & 1.9317 \\
$1 / 80$ & $5.1690 \mathrm{e}-005$ & 1.9855 & $4.1427 \mathrm{e}-005$ & 1.9697 \\
$1 / 160$ & $1.2989 \mathrm{e}-005$ & 1.9926 & $1.0459 \mathrm{e}-005$ & 1.9858 \\
\hline
\end{tabular}

Table $6 \varepsilon^{n}$ at $t=t_{n}$ for different values of $\alpha$ with $h=\frac{1}{10}$ and $\tau=\frac{1}{20}$

\begin{tabular}{lllll}
\hline $\mathrm{t}$ & $\alpha=1.1$ & $\alpha=1.5$ & $\alpha=1.95$ & $\alpha=2.0$ \\
\hline 0 & 10.087375775801739 & 9.5153933095222924 & 9.204583910317352 & 9.1860842895597257 \\
10 & 10.0873757793844356 & 9.515393313821262 & 9.204583911659553 & 9.186084292149856 \\
20 & 10.087375780023113 & 9.515393315532492 & 9.204583913085234 & 9.186084295906156 \\
30 & 10.087375780648971 & 9.515393316223834 & 9.204583914395832 & 9.186084297443383 \\
40 & 10.087375781657538 & 9.515393316456663 & 9.204583914934780 & 9.186084298335000 \\
50 & 10.087375783376320 & 9.515393317054322 & 9.204583915129753 & 9.186084299068263 \\
60 & 10.087375785301962 & 9.515393318461506 & 9.204583915590725 & 9.186084300186360 \\
70 & 10.087375786753782 & 9.515393320281280 & 9.204583917024371 & 9.186084300778976 \\
80 & 10.087375787805106 & 9.515393321510466 & 9.204583919287133 & 9.186084299627677 \\
90 & 10.087375788916159 & 9.515393321706426 & 9.204583919632452 & 9.186084299527717 \\
$100-\tau$ & 10.087375790133651 & 9.515393321480675 & 9.204583918662443 & 9.186084299808456 \\
\hline
\end{tabular}

method for implementation of the proposed difference scheme. Both theoretical analysis and numerical experiments show that the difference scheme is efficient for solving spacefractional SGE. 


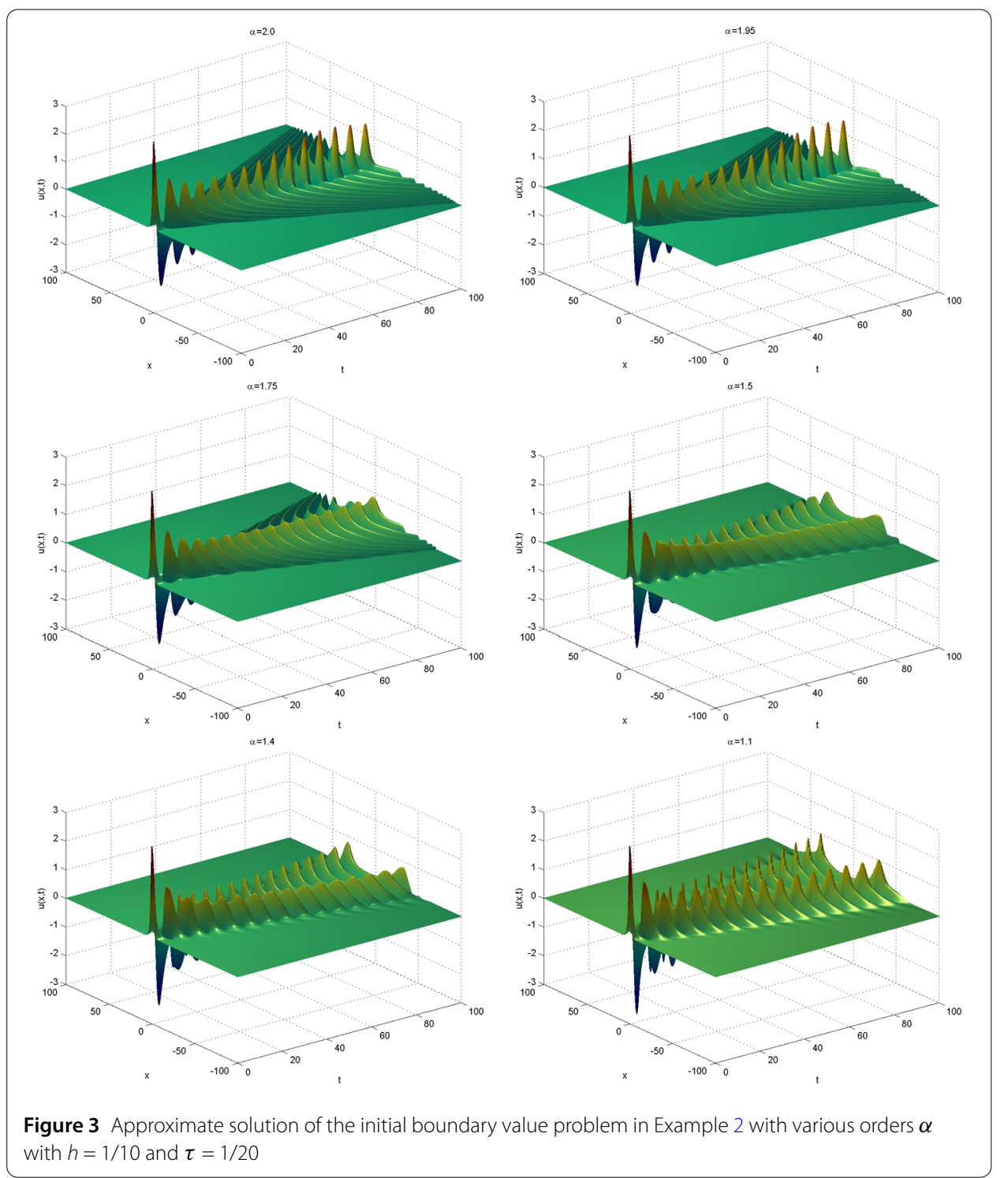

Table 7 The consumed CPU time of the regular Newton method and the revised Newton method

\begin{tabular}{ll}
\hline$h$ & CPU time of the regular Newton method \\
\hline $1 / 8$ & $18.7031 \mathrm{~s}$ \\
$1 / 16$ & $72.8281 \mathrm{~s}$ \\
$1 / 32$ & $302.9375 \mathrm{~s}$ \\
$1 / 64$ & $1.1886 \mathrm{e}+03 \mathrm{~s}$ \\
$1 / 128$ & $7.9377 \mathrm{e}+03 \mathrm{~s} \approx 2 \mathrm{~h} 12 \mathrm{~m} 18 \mathrm{~s}$ \\
\hline \hline$h$ & CPU time of the revised Newton method \\
\hline $1 / 8$ & $4.9688 \mathrm{~s}$ \\
$1 / 16$ & $14.0938 \mathrm{~s}$ \\
$1 / 32$ & $42.7500 \mathrm{~s}$ \\
$1 / 64$ & $142.3594 \mathrm{~s}$ \\
$1 / 128$ & $467.7500 \mathrm{~s} \approx 7 \mathrm{~m} \mathrm{48} \mathrm{s}$ \\
\hline
\end{tabular}

\section{Acknowledgements}

The authors would like to express the sincere thanks to the referees for their valuable comments and suggestions, which helped to improve the original paper. 


\section{Funding}

This work is supported by NSF of China (No. 11371302) and Postgraduate Innovation project of Hunan Province, China (No. CX2017B270)

\section{Competing interests}

The authors declare that they have no competing interests.

\section{Authors' contributions}

Both authors contributed equally to writing of this paper. Both authors read and approved the final manuscript.

\section{Publisher's Note}

Springer Nature remains neutral with regard to jurisdictional claims in published maps and institutional affiliations.

\section{Received: 15 December 2017 Accepted: 11 June 2018 Published online: 11 July 2018}

\section{References}

1. Ablowitz, M., Clarkson, P.: Solitons, Nonlinear Evolution Equations and Inverse Scattering. Cambridge University Press, Cambridge (1991)

2. Perring, J.K., Skyrme, T.H.: A model unified field equation. Nucl. Phys. 31, 550-555 (1962)

3. Mohebbi, A., Dehghan, M.: High-order solution of one-dimensional sine-Gordon equation using compact finite difference and DIRKN methods. Math. Comput. Model. 51, 537-549 (2010)

4. Macías-Díaz, J.: Numerical study of the process of nonlinear supratransmission in Riesz space-fractional sine-Gordon equations. Commun. Nonlinear Sci. Numer. Simul. 46, 89-102 (2017)

5. Shi, L., Hao, C., Li, C., Huang, N.: Conservation laws in sine-Gordon equation. Chin. Phys. Lett. 20, 1003-1005 (2003)

6. Zhang, F., Vázquez, L.: Two energy conserving numerical schemes for the sine-Gordon equation. Appl. Math. Comput. 45, 17-30 (1991)

7. Zhang, L., Chang, Q.: A conserving nine points finite difference scheme for the sine-Gordon equation. Math. Appl. 12, 30-35 (1999)

8. Guo, B., Pascual, P., Rodriguez, M., Vázquez, L.: Numerical solution of the sine-Gordon equation. Appl. Math. Comput. $18,1-14(1986)$

9. Jiang, C.L., Sun, J.Q., Li, H.C., Wang, Y.F.: A fourth-order AVF method for the numerical integration of sine-Gordon equation. Appl. Math. Comput. 313, 144-158 (2017)

10. Lu, X., Schmid, R.: Symplectic integration of sine-Gordon type systems. Math. Comput. Simul. 50, 255-263 (1999)

11. Voss, D.A., Khaliq, A.Q.M.: Parallel LOD methods for second order time dependent PDEs. Comput. Math. Appl. 10, 25-35 (1995)

12. Ran, M., Zhang, C.: A conservative difference scheme for solving the strongly coupled nonlinear fractional Schrödinger equations. Commun. Nonlinear Sci. Numer. Simul. 41, 64-83 (2016)

13. Wang, P., Huang, C.: An energy conservative difference scheme for the nonlinear fractional Schrödinger equations. J. Comput. Phys. 293, 238-251 (2015)

14. Guo, B., Han, Y., Xin, J.: Existence of the global smooth solution to the period boundary value problem of fractional nonlinear Schrödinger equation. Appl. Math. Comput. 204,468-477 (2008)

15. Herzallah, M., Gepreel, K.: Approximate solution to the time-space fractional cubic nonlinear Schrödinger equation. Appl. Math. Model. 36, 5678-5685 (2012)

16. Li, M., Huang, C., Wang, P.: Galerkin finite element method for nonlinear fractional Schrödinger equations. Numer. Algorithms 74, 499-525 (2017)

17. Hu, Y., Li, C., Li, H.: The finite difference method for Caputo-type parabolic equation with fractional Laplacian: one-dimensional case. Chaos Solitons Fractals 102, 361-371 (2017)

18. Bu, W., Tang, Y., Yang, J.: Galerkin finite element method for two-dimensional Riesz space fractional diffusion equations. J. Comput. Phys. 276, 26-38 (2014)

19. Jiang, Y., Ma, J.: High-order finite element methods for time-fractional partial differential equations. J. Comput. Appl. Math. 235, 3285-3290 (2011)

20. Ray, S.: A new analytical modelling for nonlocal generalized Riesz fractional sine-Gordon equation. J. King Saud Univ. Sci. 28, 48-54 (2016)

21. Alfimov, G., Pierantozzi, T., Vázquez, L.: Numerical study of a fractional sine-Gordon equation. Fract. Differ. Appl. FDA 4, 644-649 (2004)

22. Yang, Q., Liu, F., Turner, I.: Numerical methods for fractional partial differential equations with Riesz space fractional derivatives. Appl. Math. Model. 34, 200-218 (2010)

23. Sun, Z., Gao, G.: A Finite Difference Method for Fractional Differential Equations. Science Press, China (2015)

24. Kirkpatrick, K., Lenzmann, E., Staffilani, G.: On the continuum limit for discrete NLS with long-range lattice interactions. Commun. Math. Phys. 317, 563-591 (2013)

25. Wang, D., Xiao, A., Yang, W.: Maximum-norm error analysis of a difference scheme for the space fractional CNLS. Appl. Math. Comput. 257, 241-251 (2015)

26. Temam, R.: Navier-Stokes Equations: Theory and Numerical Analysis. North-Holland, Amsterdam (1977) 